\title{
Improving the limit of detection for Sanger sequencing: A comparison of methodologies for KRAS variant detection
}

Colin J. Davidson ${ }^{1}$, Emily Zeringer ${ }^{4}$, Kristen J. Champion ${ }^{2}$, Marie-Pierre Gauthier ${ }^{1}$, Fawn Wang ${ }^{1}$, Jerry Boonyaratanakornkit ${ }^{3}$, Julie R. Jones ${ }^{2}$, and Edgar Schreiber ${ }^{1}$

${ }^{1}$ Life Technologies, Foster City, CA, USA, ${ }^{2}$ Greenwood Genetic Center, Greenwood, SC, USA, ${ }^{3}$ Life Technologies, Benicia, CA, USA, and ${ }^{4}$ Life Technologies, Austin, TX, USA

Fluorescent dye terminator Sanger sequencing (FTSS), with detection by automated capillary electrophoresis (CE), has long been regarded as the gold standard for variant detection. However, software analysis and basecalling algorithms used to detect mutations were largely optimized for resequencing applications in which different alleles were expected as heterozygous mixtures of 50\%. Increasingly, the requirements for variant detection are an analytic sensitivity for minor alleles of $<20 \%$, in particular, when assessing the mutational status of heterogeneous tumor samples. Here, we describe a simple modification to the FTSS workflow that improves the limit of detection of cell-line gDNA mixtures from 50\%-20\% to 5\% for G>A transitions and from 50\%-5\% to 5\% for $\mathrm{G}>\mathrm{C}$ and $\mathrm{G}>\mathrm{T}$ transversions. In addition, we use two different sample types to compare the limit of detection of sequence variants in codons 12 and 13 of the KRAS gene between Sanger sequencing and other methodologies including shifted termination assay (STA) detection, single-base extension (SBE), pyrosequencing (PS), high- resolution melt (HRM), and real-time PCR (qPCR).

Keywords: Sanger sequencing; capillary electrophoresis; variant detection; KRAS; fragment analysis; genotyping; mutation detection

The KRAS (v-Ki-ras2 Kirsten rat sarcoma viral oncogene homolog) gene encodes a GTPase binding protein that functions as an intermediary in the RAS-MAPK pathway, one of several signaling cascades downstream of epidermal growth factor receptor (EGFR) activation (1). EGFR signaling pathways are important in the development and progression of several aggressive cancers, with KRAS gene mutations common in pancreatic, lung, and colorectal cancers (2). Activating KRAS mutations, most frequently described in codons 12 and 13 , result in a constitutively activated form of KRAS causing EGFR pathway signaling independent of EGFR activation, thus making therapeutic agents that block EGFR, such as cetuximab or panitumumab, ineffective $(3,4)$. Consequently, anti-EGFR therapies are recom- mended only for patients with wild-type $K R A S$ status $(5,6)$. Although not limited to, a typical analysis of $K R A S$ mutational status would be performed on biopsies of tumor tissue that are processed into formaldehyde fixed and paraffin embedded (FFPE) blocks, from which sections are generated and gDNA extracted. As a consequence of the fixation process, the quality of gDNA from these samples is compromised and, additionally, the samples can be highly heterogeneous with a mixture of normal cells as well as both $K R A S$ mutant and non-mutant tumor cells. Therefore, the methods used to determine the mutational status of the KRAS gene must be both sensitive and robust.

A number of platforms and techniques can be used to assess the mutation status of the KRAS gene with fluorescent dye terminator Sanger sequencing (FTSS) being widely acknowledged as the standard for direct detection of sequence variants. However, the software analysis and basecalling algorithms used to detect mixed bases, a heterozygous state at a particular nucleotide, were designed for typical resequencing mutation detection in which different alleles are expected as heterozygous mixtures of $50 \%$ and, as a result, do not facilitate sensitive detection of minor alleles from heterogeneous samples.

In this paper, we present a simple method for improving the analytic sensitivity of FTSS reactions. A standard cycle sequencing workflow is followed consisting of PCR amplification, followed by cycle sequencing using the BigDye Terminator v1.1 Cycle Sequencing Kit. The reaction is first analyzed by capillary electrophoresis

\section{Method summary:}

This study describes a simple extension of the Sanger sequencing workflow for improving the detection of minor component alleles. The novelty of the approach is the addition of a size standard to the completed sequencing reaction and the collection of sequencing data as a fragment file for analysis using genotyping software. Sizing analysis facilitates detection by separating the minor component alleles in two dimensions (by size and dye color) from fragments corresponding to the major component allele. Further, genotyping software allows user control over peak detection, such as setting a peak height detection threshold so that minor component alleles can be readily distinguished from baseline noise. 
(CE) and a standard sequencing file (.abl) is generated. Following collection of the sequencing file, a fluorescently labeled size standard is added to the sequencing reaction. Then, the sequencing reaction is analyzed by $\mathrm{CE}$ as a fragment analysis reaction generating a standard fragment file (.fsa). The addition of the size standard allows for the sizing of all the fluorescently labeled sequencing reaction fragments. The sized fragments then can be further analyzed with genotyping software, which allows automated allele calling through the use of bins specifying the expected size and dye of a particular nucleotide fragment. The use of genotyping software allows the user greater control over how the raw data are analyzed including control over the peak detection algorithm, a functionality that is not possible with base-calling analysis of sequencing sample files (.ab1). To benchmark the improvements to CE FTSS, the following detection methods were examined: shifted termination assay (STA) (7), single-base extension (SBE) $(8,9)$, pyrosequencing (PS) (10), high-resolution melt (HRM) (11), and real-time PCR (qPCR) (12).

\section{Materials and methods}

\section{Samples}

Sample Type 1: Various percentages $(50 \%$, $20 \%, 10 \%, 5 \%, 2 \%, 1 \%$, and $0.5 \%$ ) of gDNA extracted from KRAS mutant cancer celllines were mixed with gDNA extracted from a $K R A S$ wild-type cancer cell line (HT-29). The KRAS mutant cancer celllines were: A549 (c.34G>A), MIA-PaCa-2 (c.34G>T), SW 1116 (c.35G>C); SW480 (c.35G>T), and HCT116 (c.38G>A).

Sample Type 2: Various percentages (20\%, $10 \%, 5 \%, 2 \%, 1 \%)$ of KRAS mutant cells mixed with wild-type cells as well as $100 \%$ wild-type and mutant $B R A F$ cells were formaldehyde fixed and paraffin embedded (FFPE) to create a cell block and sections (AcroMetrix, Benicia, CA, USA) from which gDNA was extracted.

\section{Sample preparation}

$B R A F$ FFPE sample gDNA was extracted from each section using the MagMAX FFPE DNA Isolation Kit (Ambion, Austin, TX, USA). All other total gDNA was extracted using RecoverAll (Ambion) according to the manufacturer's instructions. The gDNA then was quantified by OD $260 \mathrm{~nm}$ using the NanoDrop ND-1000 Spectrophotometer (Thermo Scientific, Wilmington, DE, USA) and the Quantifiler Human DNA Quantification Kit (Applied Biosystems, Foster City, CA, USA) using the 7500 Real-Time PCR System (Applied Biosystems).

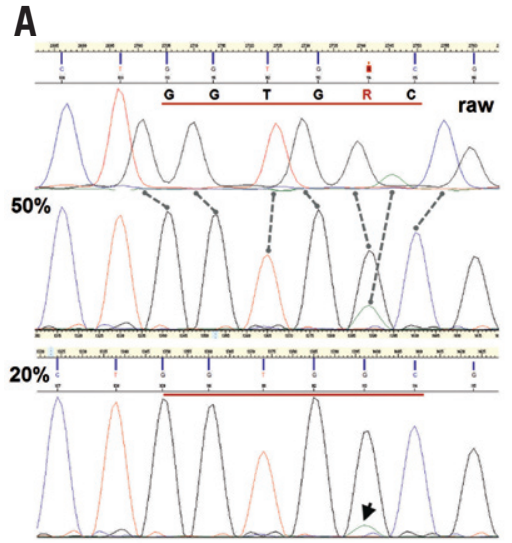

B

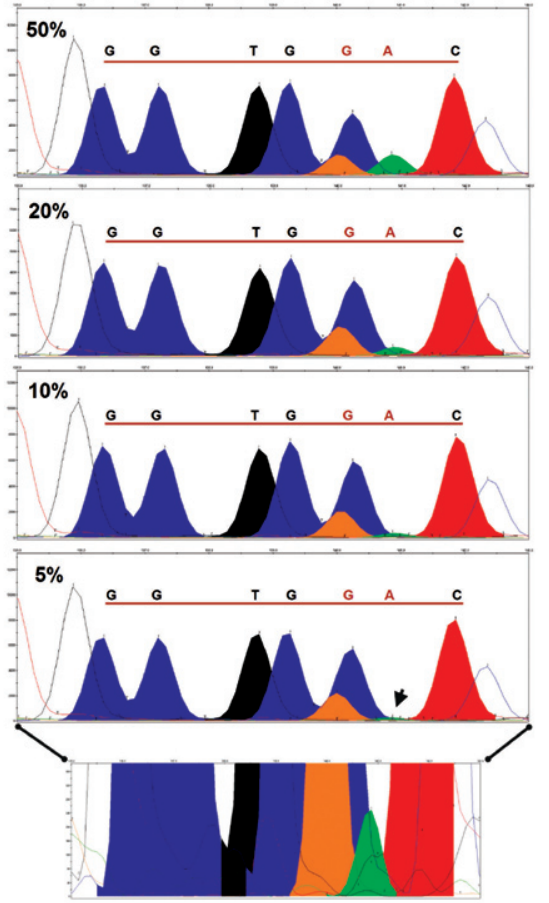

Figure 1. Comparison of sequencing reaction electropherograms derived from the analysis of KRAS wild type and c.38G $>$ A (p.G13D) mutant gDNA mixtures of $50 \%, 20 \%, 10 \%, 5 \%$ (mutant/wild type). Electropherograms on the left were analyzed as sequencing reactions on a $3500 x \mathrm{~L}$ using sequencing software, while electropherograms on the right are the same reactions to which a size standard was added and the sequencing reaction was analyzed as fragment analysis reaction on a $3500 \mathrm{xL}$ followed by analysis using GeneMapper v4.1 software. (A) The 50\% mutant/wild-type mixture electropherogram shows the raw trace pattern on top and the analyzed and basecalled trace beneath. Note that the raw fragments are mobility shifted relative to the analyzed fragments as indicated by the dotted gray lines. The red line highlights codon 12 (GGT) and codon 13 (GGC) of the KRAS gene with the $c .38 \mathrm{G}>\mathrm{A}$ mutation indicated by the mixed base designation $(R)$. The mutant fragment can still be reliably detected in the $20 \%$ mixture analyzed trace (black arrow), however, in the analyzed traces from the $10 \%$ and $5 \%$ mixtures, the fragment is indistinguishable from the baseline noise. (B) Electropherograms of the same mixtures analyzed using GeneMapper genotyping software. Note that Applied Biosystems sequencing software designates fragments labeled with ddGTP - black (yellow), ddATP - green, ddTTP - red, and ddCTP - blue; whereas, genotyping software such as GeneMapper displays fragments labeled with ddGTP - blue, ddATP - green, ddTTP - black (yellow), ddCTP red, and LIZ-labeled size standard fragments are orange. The red line indicates codon 12 (GGT) and codon 13 (GGC) of the KRAS gene with the c.38G>A mutation highlighted in red. Below the electropherogram of the $5 \%$ mixture is a magnification of the $Y$-axis showing the region of interest that illustrates the green c.38G $>$ A mutant fragment (black arrow) can be readily distinguished from baseline noise.

Type 1 samples were prepared by diluting gDNA from KRAS mutant cell-lines into wild-type $K R A S$ cell-line (HT-29) to a final concentration of $100 \mathrm{ng} / \mu \mathrm{L}$.

\section{Fluorescent dye terminator Sanger sequencing}

PCR primer pairs (5'-tgtaaaacgacggccagtTATTTGATAGTGTATTAACCTTATGTGTG-3' and 5'-caggaaacagctatgaccGAAACCTTTATCTGTATCAAAGAATG-3') and (5'-tgtaaaacgacggccagtGCTTGCTCTGATAGGAAAATGAGATC-3' and 5'-caggaaacagctatgaccATCCAGACAACTGTTCAAACTGATG-3') with M13 tails (lower case letters) were used respectively to amplify exon 2 of the $K R A S$ gene and exon
15 of the $B R A F$ gene. Each PCR reaction contained $1 \times$ AmpliTaq Gold Fast PCR Master Mix UP (Applied Biosystems, Foster City, CA, USA), $10 \mu \mathrm{M}$ primers, and $100 \mathrm{ng} /$ $\mu \mathrm{L}$ of template gDNA in $30 \mu \mathrm{L}$ final volume. Cycling conditions performed on the Veriti Thermal Cycler (Applied Biosystems, Foster City, CA, USA) were: $95^{\circ} \mathrm{C} 10 \mathrm{~min}, 35 \times$ $\left(96^{\circ} \mathrm{C}-3 \mathrm{~s}, 62^{\circ} \mathrm{C}-3 \mathrm{~s}, 68^{\circ} \mathrm{C}-15 \mathrm{~s}\right), 72^{\circ} \mathrm{C}$ $10 \mathrm{~s}$. Following amplification, the reaction was treated with ExoSAP-IT enzyme (USB Corporation, Cleveland, OH, USA) to remove unincorporated primers and dNTPs. Cycle sequencing was performed on the Veriti Thermal Cycler with reaction containing $1 \times$ BigDye Terminator Cycle Sequencing Kit vl.1 (Applied Biosystems, Foster City, CA, USA), 3.2 $2 \mu \mathrm{MM} 13$ primer, and $2 \mu \mathrm{L}$ of PCR 
A

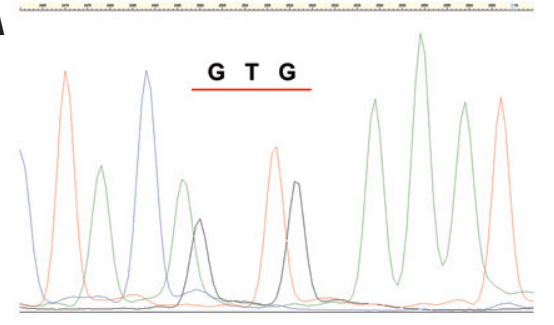

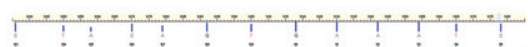
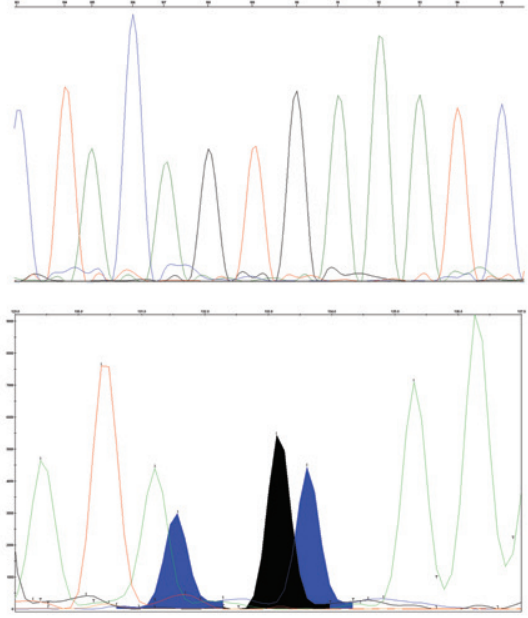

B

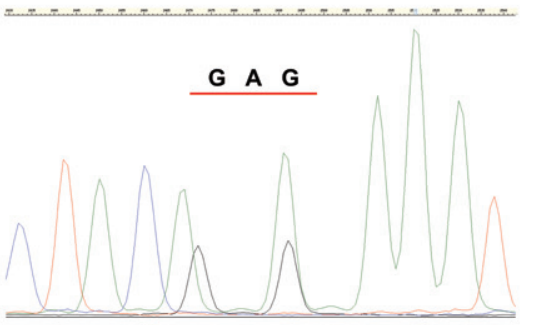

$\ldots \ldots \ldots \ldots \ldots \ldots \ldots \ldots+\ldots \ldots$
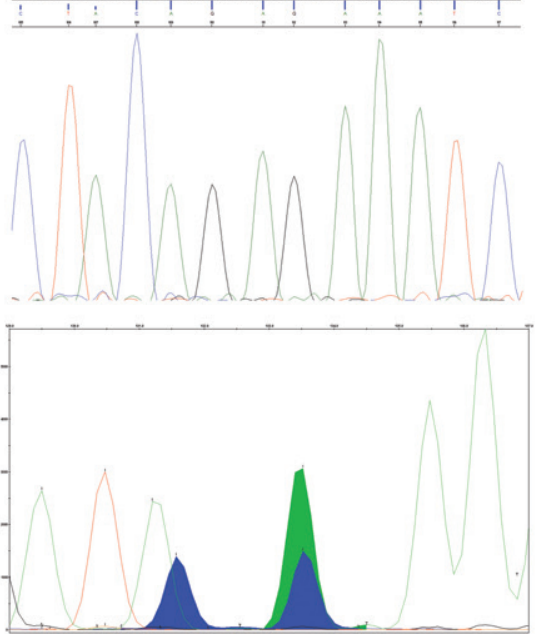

C

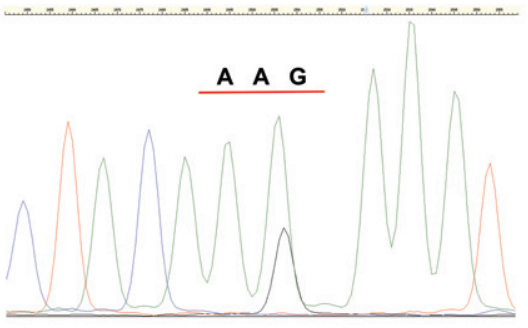

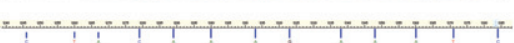
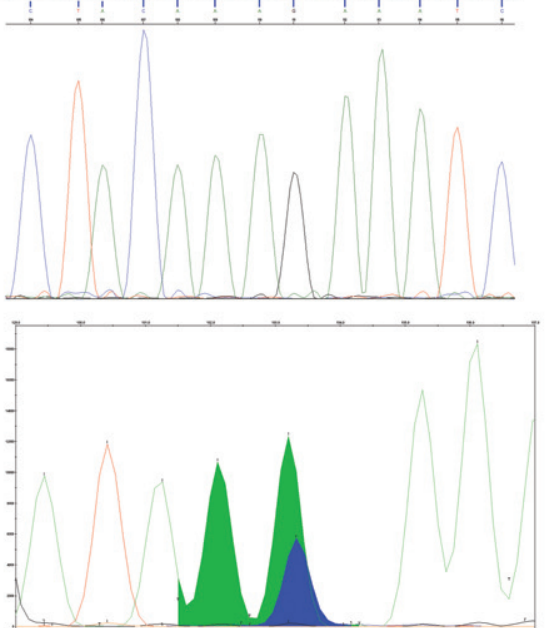

Figure 2. Comparison of sequencing reaction electropherograms derived from the analysis of BRAF wild type, c.1799T>A (p.V600E) and c.1798_1799GT>AA

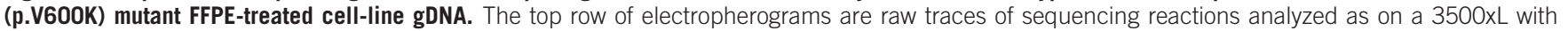

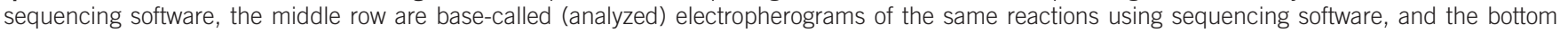

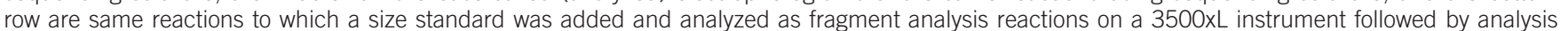

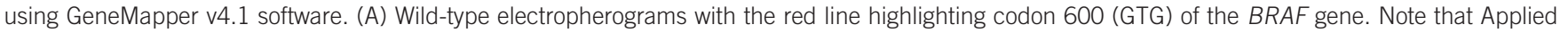

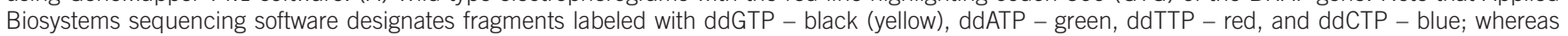

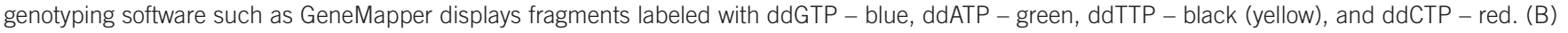

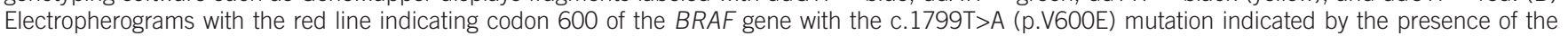

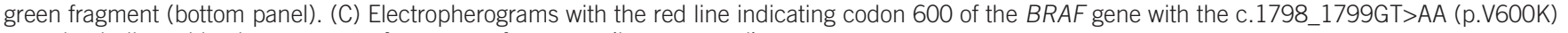
mutation indicated by the presence of two green fragments (bottom panel).

product in a $10 \mu \mathrm{L}$ final volume. Cycling conditions were: $96^{\circ} \mathrm{C}-1 \mathrm{~min}, 25 \times\left(96^{\circ} \mathrm{C}\right.$ $\left.10 \mathrm{~s}, 50^{\circ} \mathrm{C}-5 \mathrm{~s}, 60^{\circ} \mathrm{C}-75 \mathrm{~s}\right), 72^{\circ} \mathrm{C}-10 \mathrm{~s}$. Cycle sequencing reaction products were purified using Centri-Sep spin columns (Applied Biosystems). Purified reactions were vacuum concentrated and the samples reconstituted with $10 \mu \mathrm{L}$ of Hi-Di Formamide (Applied Biosystems). Capillary electrophoresis was performed using the $3500 \times \mathrm{xL}$ Genetic Analyzer capillary electrophoresis system (Applied Biosystems) with POP-7 polymer and a $50 \mathrm{~cm}$ array length. The instrument protocol used was the RapidSeq50_POP7 run module in combination with the $\mathrm{E}$ dye set. Sequences were aligned to a reference sequence using Variant Reporter Software v1.1 (Applied Biosystems) and variants assessed by visual inspection.

Fragment analysis of Sanger sequencing (FASS) reactions

For FASS reactions, $0.5 \mu \mathrm{L}$ of GeneScan600LIZ v2.0 size standard (Applied Biosystems) was added to purified cycle sequencing reaction products following collection of sequencing sample files (.ab1). Capillary electrophoresis was performed using the $3500 \mathrm{xL}$ Genetic Analyzer capillary electrophoresis system with POP-7 polymer and a $50 \mathrm{~cm}$ array length. The instrument protocol used was the FragmentAnalysis50_POP7 run module in combination with the E5 dye set. GeneMapper v4.1 Software (Applied Biosystems) was used for sizing of fragments and genotyping.

\section{Shifted termination assay}

PCR amplification and STA reactions were performed on the 9700 Thermal Cycler (Applied Biosystems). Samples were assessed using the KRAS Mutational Analysis Reagents (Applied Biosystems) according to the manufacturer's protocol. Capillary electrophoresis was performed using the 3500xL Genetic Analyzer capillary electrophoresis system with POP-7 polymer and a $50 \mathrm{~cm}$ array length. The electrophoresis conditions for c.35G $>\mathrm{T}$ (p.Gly12Val) variants used POP-6 polymer and a $50 \mathrm{~cm}$ array length on the $3500 x \mathrm{~L}$ Genetic Analyzer. GeneMapper v4.1 Software was used for sizing of fragments and genotyping.

\section{Single-base extension}

Single-base extension reaction contained: 1X SNaPshot kit (Applied Biosystems), $0.2 \mu \mathrm{M}$ of multiplexed primers (KRASc34 - 5'-AACTTGTGGTAGTTGGAGCT-3', KRASc35 - MM-5'ACTTGTG-GTAGTTGGAGCTG-3', KRASc37 - MM-5'-GTGGTAGTTGGAGCTGGT-3', KRASc38 - MM-5'GTGGTAGTTGGAGCTGGTG-3' $(\mathrm{MM}=$ mobility modified $)$, and $3 \mu \mathrm{L}$ of PCR product (from FTSS reaction) in a $10 \mu \mathrm{L}$ final volume. After treatment with $2 \mathrm{U}$ of shrimp alkaline phosphatase (USB Corporation), $0.5 \mu \mathrm{L}$ of SBE products was combined with $9 \mu \mathrm{L}$ of Hi-Di Formamide and $0.5 \mu \mathrm{L}$ of GeneScan-120LIZ size standard (Applied Biosystems). Following denaturation at $95^{\circ} \mathrm{C}$ for $2 \mathrm{~min}$, capillary electrophoresis was performed using the 
3500xL Genetic Analyzer capillary electrophoresis system with POP-7 polymer and a $50 \mathrm{~cm}$ array length. GeneMapper v 4.1 Software was used for sizing of fragments and genotyping.

\section{Pyrosequencing}

Samples were amplified and sequenced in triplicate using the KRAS Pyro Kit (Qiagen, Valencia, CA, USA) according to the manufacturer's recommendation and the reactions were analyzed on the PyroMark Q24 instrument (Qiagen). The percentage of mutant allele was calculated by the PyroMark Q24 software (Qiagen).

\section{High-resolution melt}

Samples were amplified using MeltDoctor HRM Master Mix (Applied Biosystems) according to the manufacturer's recommendation and run on Viia 7 Real-Time PCR System (Applied Biosystems) with default HRM setting and an annealing temperature of $67^{\circ} \mathrm{C}$.

\section{Real-time PCR}

Samples were PCR amplified using the DxS K-RAS Mutation Kit (Qiagen) according to the manufacturer's recommendation and the reactions were analyzed on the 7500 Real-Time PCR System.

\section{Copy number variation}

Copy number variation (CNV) was assessed for samples using 19 pre-designed TaqMan CNV assays (Applied Biosystems) distributed across the KRAS gene and the reactions were analyzed along with wild-type calibrator samples and reference assays (RNaseP and TERT) using the TaqMan Genotyping Master Mix (Applied Biosystems) according to manufacturer's protocol with the reactions analyzed on the 7500 Real-time PCR System.

\section{Results and discussion}

The analysis of a Sanger sequencing reactions as fragment analysis reactions (FASS) is possible on Applied Biosystems Genetic Analyzers because of the spectral compatibility of the E dye set and the E5 dye set, used to spectrally calibrate the four fluorophores in the BigDye Terminator v1.1 chemistry and the five fluorophores of the dRhodamine chemistry (DS-02 dye set: dR110, dRGG, dTAMRA, dROX, LIZ), respectively. Applied Biosystems Genetic Analyzers require a spectral calibration, a deconvolution matrix that compensates for fluorophore overlap (reduces raw data from the instrument) in the 4 dye or 5 dye data stored in each sample file. The fifth dye (LIZ) of the E5 dye set is used to label the size standard, which comprises labeled DNA fragments of known sizes that are added to the sample prior to electrophoresis. The genotyping software assigns the defined size values to the appropriate peaks of the size standard and, by means of a size calling algorithm, the sizes of all unknown fragments are determined based on the known sizes of the internal size standard. Use of internal size standard results in precise molecular length determination since the internal size standard fragments and the unknown sequencing reaction fragments undergo exactly the same electrophoretic forces thus compensating for injection-to-injection variation on CE instruments. With precise sizing, it is possible to identify alleles based on bin definitions for a particular nucleotide position of interest. A bin is a defined basepair size range and dye color for a particular allele fragment (Supplementary Figure S1).

To define the bin positions for the wild-type and mutant alleles of the KRAS gene, the appropriate fragments must be visually identified in the raw data by extrapolation from the analyzed (base-called) sequencing electropherogram (Figure 1A). This can be accomplished by opening a sequencing trace file using the free software Sequence Scanner v1.0 (www.lifetechnologies.com) and selecting the analyzed and raw view tab. Note that the fragments in the analyzed sequencing electropherogram will be shifted relative to the raw view because of the base-calling algorithm adjusting fragments due to mobility differences of the dye terminators (Figure 1A, top panel). The sequencing raw view electropherogram pattern can then be located in the plot view of the genotyping software (compare raw view of Figure $1 \mathrm{~A}$ with top panel of Figure 1B). To analyze bi-directional sequencing reactions, two panel and bin sets were created, one for M13 forward reactions and a second for M13 reverse reactions (Supplementary Figure S1).

Analysis of a sequencing reaction as a fragment file (.fsa) also benefits from the greater user control over peak detection that is possible with genotyping software compared with sequencing software. In addition to automated allele detection by fragment size and dye color, the advanced peak detection algorithm in GeneMapper software allows user control over peak amplitude thresholds that can be set individually for each dye color. This allows the user to determine the peak height at which the software detects peaks of interest but eliminates noise with the software reporting to the user only those peaks with heights that are at least the peak amplitude threshold for that dye. Additional parameters, such as polynomial degree and peak window size, can be used to adjust the sensitivity of peak detection. It is advisable that the above parameters be empirically determined to optimize the peak detection sensitivity for a particular sequencing reaction.

Using purified gDNA mixtures extracted from $K R A S$ mutant and wild-type cell-lines (sample type 1), the limit of detection was improved to $5 \%$ for the detection of $G>A$ transitions (c.34G>A, p.G12S; c.38G>A, p.G13D) as well as for $G>T$ (c.34G $>T$, p.G12C; c.35G $>$ T, p.G12V) and $G>C$ transversions (c.35G>C, p.G12A), when the fragment approach (FASS) to the analysis of a FTSS reactions was used (Table 1 and Supplementary Table S2). This is compared with a limit of detection of $50 \%$ via software detection and $20 \%$ by visual inspection when the same $G>A$ transition mutations were analyzed via sequencing software. For $\mathrm{G}>\mathrm{T}$ and $\mathrm{G}>\mathrm{C}$ transversions, the analytic sensitivity for FTSS was $50 \%-20 \%$ by software detection and $20 \%-5 \%$ via visual inspection. It is worth noting that the limit of detection for variant c.35G $>\mathrm{T}$ (p.G12V) was unchanged at $5 \%$ whether analyzed by visual inspection as a sequencing reaction or as a FASS reaction. The improved detection of the sequencing reaction for this variant is likely due to a combination of zygosity and copy number effects increasing the concentration of the mutant allele since the KRAS mutant cell-line used for c.35G $>\mathrm{T}$ (p.G12V) mixtures was found to be homozygous for c. $35 \mathrm{G}>\mathrm{T}$ mutant with a copy number of 3 (Supplementary Table S1). It is also worth pointing out that the detection of variant c.38G $>\mathrm{A}$ (p.G13D) is more challenging since the cell-line that was used was heterozygous with a normal copy number of two (Supplementary Table S1). As a consequence, the actual concentration of mutant allele in the mixture was half the expected amount which was consistent with the calculated percent mutant allele detected by the PS assay (Table 1).

When analyzed as a fragment analysis reaction, the analytic sensitivity of FTSS improves to a level that is comparable to the PS and HRM approaches for the same mutations (Table 1 and Supplementary Table S2). For G>A transitions, the limit of detection was found to be $10 \%-2 \%$ and $20 \%-10 \%$ for the PS and HRM assays, respectively. The limit of detection observed in this study for cell-line mixtures of the c.38G $>$ A (p.G13D) variant is similar to the previously reported values of $15 \%-20 \%, 5 \%$, and $10 \%$ for FTSS, PS, and HRM, respectively (13). For G $>\mathrm{T}$ transversions, the analytic sensitivity of PS and HRM assays was $5 \%$ with the PS assay demonstrating a 
Table 1. Summary results for gDNA mixtures of KRAS mutant and wild-type cancer cell-lines (sample type 1).

\begin{tabular}{|c|c|c|c|c|c|c|c|c|}
\hline Assay & Variant & $50 \%$ (MUT/WT) & $20 \%$ (MUT/WT) & $10 \%$ (MUT/WT) & $5 \%$ (MUT/WT) & $2 \%$ (MUT/WT) & $1 \%$ (MUT/WT) & $0.5 \%$ (MUT/WT) \\
\hline \multirow[b]{2}{*}{ FTSS } & c. $34 \mathrm{G}>\mathrm{T}, \mathrm{p} . \mathrm{G} 12 \mathrm{C}$ & + & $+/-$ & $+/-^{*}$ & $-*$ & - & - & - \\
\hline & c. $35 \mathrm{G}>\mathrm{T}, \mathrm{p} . \mathrm{G} 12 \mathrm{~V}$ & + & + & $+/-$ & $+/-*$ & -* & - & - \\
\hline \multirow[b]{2}{*}{ FASS } & c. $34 \mathrm{G}>\mathrm{T}, \mathrm{p} . \mathrm{G} 12 \mathrm{C}$ & + & + & + & $+*$ & $-*$ & - & - \\
\hline & c. $35 \mathrm{G}>\mathrm{T}, \mathrm{p} . \mathrm{G} 12 \mathrm{~V}$ & + & + & + & $+*$ & $-*$ & - & - \\
\hline \multirow{3}{*}{ HRM } & c. $34 \mathrm{G}>\mathrm{T}, \mathrm{p} . \mathrm{G} 12 \mathrm{C}$ & + & + & + & $+^{*}$ & $-*$ & - & - \\
\hline & c. $35 \mathrm{G}>\mathrm{T}$, p.G12V & + & + & + & $+^{*}$ & $-*$ & - & - \\
\hline & c. $38 \mathrm{G}>\mathrm{A}, \mathrm{pG} 13 \mathrm{D}$ & + & + & $+^{*}$ & $-*$ & - & - & - \\
\hline PS & c. $38 \mathrm{G}>\mathrm{A}, \mathrm{pG} 13 \mathrm{D}$ & $+(28)$ & $+(13)$ & $+(4)^{*}$ & $-*$ & - & - & - \\
\hline \multirow{3}{*}{ SBE } & c. $34 \mathrm{G}>\mathrm{T}, \mathrm{p} . \mathrm{G} 12 \mathrm{C}$ & + & + & + & + & + & $+*$ & $-*$ \\
\hline & c. $35 \mathrm{G}>\mathrm{T}, \mathrm{p} . \mathrm{G} 12 \mathrm{~V}$ & + & + & + & + & + & $+*$ & $+*$ \\
\hline & c. $38 \mathrm{G}>\mathrm{A}, \mathrm{pG} 13 \mathrm{D}$ & + & + & + & + & + & $+*$ & -* \\
\hline \multirow{3}{*}{ STA } & c.34G>T, p.G12C & + & + & + & + & + & $+*$ & $-*$ \\
\hline & c. $35 \mathrm{G}>\mathrm{T}, \mathrm{p} . \mathrm{G} 12 \mathrm{~V}$ & + & + & + & + & + & $+*$ & $+*$ \\
\hline & c. $38 \mathrm{G}>\mathrm{A}, \mathrm{pG} 13 \mathrm{D}$ & + & + & + & + & + & $+{ }^{*}$ & $-*$ \\
\hline \multirow[b]{2}{*}{ qPCR } & c. $34 \mathrm{G}>\mathrm{T}, \mathrm{p} . \mathrm{G} 12 \mathrm{C}$ & NA & + & + & + & + & $+^{*}$ & $-^{*}$ \\
\hline & c.35G>T, p.G12V & NA & + & + & + & + & $+^{*}$ & $+^{*}$ \\
\hline
\end{tabular}

$5 \%$ limit of detection for the $\mathrm{G}>\mathrm{C}$ transversion mutant c.35>C (p.G12A).

The FASS, PS, and HRM approaches constitute a group of assays with equivalent analytic sensitivity $(10 \%-5 \%)$; a second grouping of more sensitive assays (2\%-1\%) consists of the SBE, STA, and qPCR assays. The limit of detection for a $G>A$ transition mutation (c.38G>A, p.G13D) was found to be $1 \%$ for SBE, STA, and qPCR (Table 1). The $1 \%$ analytical sensitivity for $G>A$ transition mutations was confirmed for the SBE and STA assays using variant c.34G>A (p.G12S) (Supplementary Table S2). An analytical sensitivity of $1 \%-0.5 \%$ was observed for SBE, STA, and qPCR assay applications for $\mathrm{G}>\mathrm{T}$ transversion mutations. As indicated above, achieving a limit of detection of $0.5 \%$ is an overestimate since the mutant cell line used for the c.35G $>$ T (p.G12V) mixtures was found to be homozygous for the mutant allele with a copy number of 3 (Supplementary Table S1), thus the actual percentage of mutant allele in the mixture is $>0.5 \%$. The $\mathrm{G}>\mathrm{C}$ transversion variant, c.35G>C (p.G12A), was found to be $2 \%$ and $1 \%$, for the SBE and STA assays, respectively (Supplementary Table S2).

The second sample type used in this study, sample type 2, was FFPE mixtures of various percentages of $K R A S$ mutant and wild-type cells from which gDNA was extracted prior to $K R A S$ variant detection. A comparison of the results for the same mutation between sample type 1 (Table 1) and sample type 2 (Table 2) gives an indication of the robustness of the methodology used to detect that mutation since the fixation process used in the creation of FFPE blocks adversely influences the integrity of the gDNA in the sample. Again, the assays distributed into two groups, with FTSS, FASS, PS, and HRM demonstrating limit of detection between 20\%-5\% depending on the variant while the SBE, STA and qPCR assays were found to have analytic sensitivities ranging between 5\%-1\%. For the $G>A$ transition mutation variant (c.38G >A, p.G13D), the limit of detection was improved from $20 \%$ by visual inspection of the sequencing electropherogram to $10 \%$ when the FASS approach was used (Table 2 ). For this type of mutation the effect of sample source resulted in a decline in the analytic sensitivity for the FASS approach from $5 \%$ for this variant in the sample type 1 mixtures. For $\mathrm{G}>\mathrm{A}$ transition variant c.38G $>$ A (p.G13D), the analytic sensitivity dramatically declined for the PS assay to $20 \%$ (from $5 \%$ for sample type 1 ) and $20 \%$ for HRM (from $10 \%$ for sample type 1). Similar to the other methods investigated the analytic sensitivity for the $G>A$ transition variant c.38G $>$ A (p.G13D) decreased the most, with the STA and the qPCR assays detecting the 5\% mixture (compared with $1 \%$ for sample type 1 ) and the SBE assay detecting the $2 \%$ mixture (versus $1 \%$ for sample type 1 ).

For FFPE mixtures, the limit of detection for FTSS was improved for G>T transversion mutations from $20 \%-10 \%$ by visual inspection to $10 \%-5 \%$ when analyzed by the FASS approach. The analytic sensi- 
Table 2. Summary results for FFPE mixtures of KRAS mutant and wild-type cancer cell-lines (sample type 2).

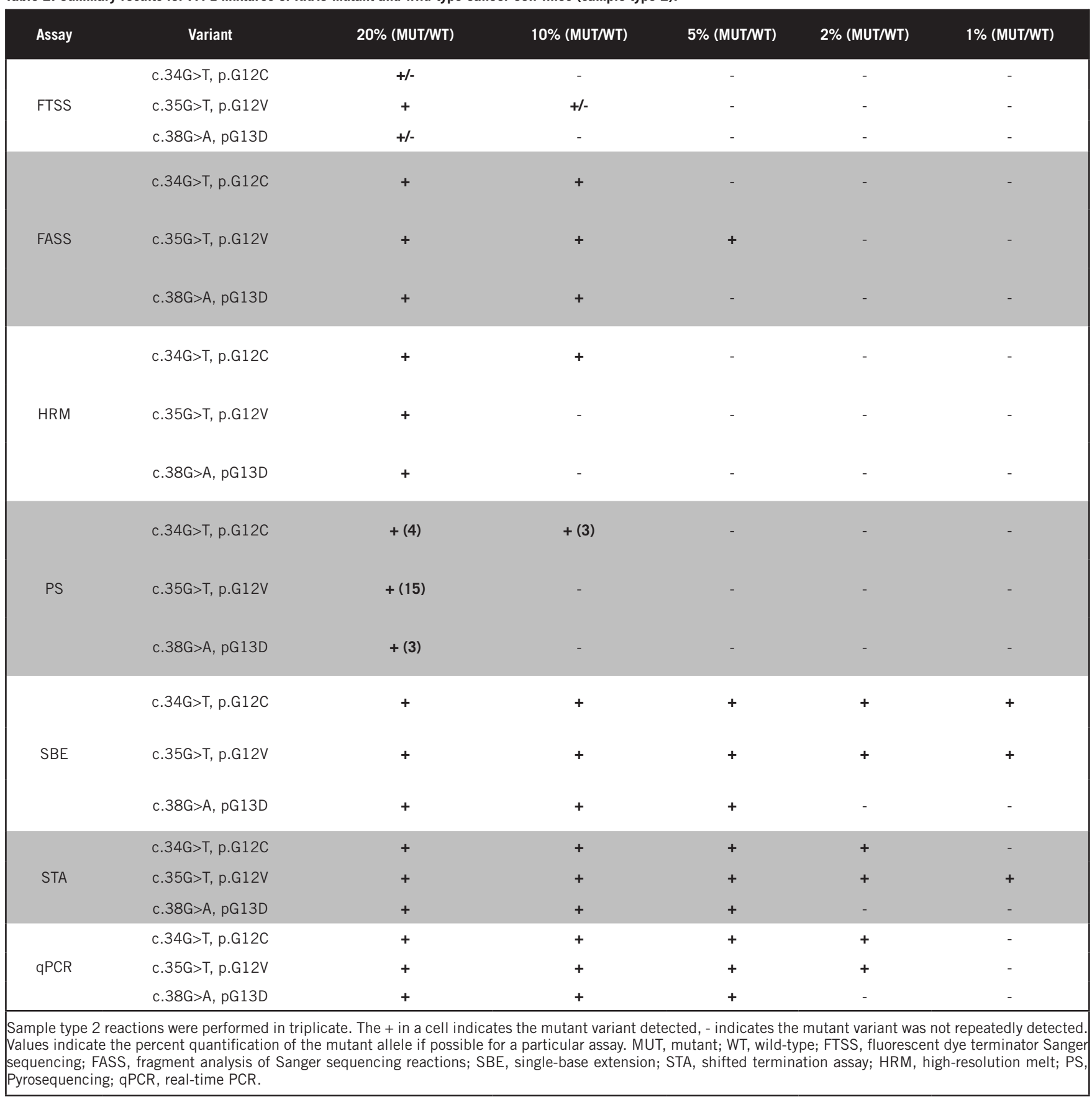

tivity for variant c.34G>T (p.G12C) declined from $5 \%$ compared with cell-line gDNA mixtures (sample type 1) to $10 \%$ for sample type 2 but was unchanged for variant c.35G $>\mathrm{T}$ (p.G12V). The limit of detection and influence of the FFPE sample source was identical for the PS and HRM assays (10\% sample type 2 versus $5 \%$ sample type 1) for variant c.34G $>T$ (p.G12C). More marked declines were observed for variant c. $35 \mathrm{G}>\mathrm{T}$ (p.G12V) using sample type 2 with the limit of detection for both the PS and HRM assays determined to be $20 \%$ versus $5 \%$ for sample type 1 . The influence of the FFPE sample source did not impact the limit of detection for variant c.34G $>\mathrm{T}$ (p.G12C) for SBE while the analytic sensitivity declined for both STA and qPCR assays (2\% sample type 2 versus $1 \%$ sample type 1 ). The analytic sensitivity of the qPCR assay was $2 \%$ for sample type 2 (compared with $0.5 \%$ for sample type 1 ) for variant c.35G $>$ T (p.G12V), while the limit of detection using sample type 2 for SBE and the STA assays was 1\% (compared with $0.5 \%$ for sample type 1).

To demonstrate that the FASS approach is amenable to the detection of both simple and complex substitution/ missense mutations, FFPE treated cell lines (sample type 2) that are wild-type and with mutations in codon 600 of the $B R A F$ gene were investigated (Figure $2)$. The FASS method can be applied to the detection of single point mutation, c.1799T $>\mathrm{A}$, as well as double point mutation, c. 1798 1799GT>AA. The precise sizing possible on CE instruments due to the addition of an internal size standard facilitates the identification of variant fragments and differentiates the mutant alleles from wild fragments since 
the mutant and wild type fragments are separated in two dimensions - by size and dye color. Precise sizing coupled with analysis by genotyping software allows reproducible identification of variants by strictly defining a bin (specifies the dye color and size range of $<1 b p$ for a fragment) for mutant alleles versus bins for the identification of wild-type alleles. The dimension of size separation is lost when sequencing reactions are analyzed by sequencing analysis soft ware since the base calling algorithms compensate for mobility differences of the fragments so that minor component variant peaks are represented under the fragment peak of the wild-type allele. Further, unlike genotyping software, sequencing analysis software does not allow user control over peak detection such as setting a peak height detection threshold so that variant fragments from minor component alleles can be readily distinguished from the baseline noise.

In summary, it is important to consider the influence of the sample (FFPE versus purified gDNA), copy number, and zygosity when determining the limit of detection for a method or assay. Although the relevance of low levels of $K R A S$ mutation is unknown, potential tumor heterogeneity resulting in samples that may contain a low percentage of mutant cells necessitates the need for analytically sensitive and robust methods such as SBE, STA, and qPCR. However, CE-based FTSS has several advantages over the other assays investigated here, in that the technique is not target specific but with proper primer design all nucleotides within a region of interest can be interrogated. Similarly, the sequencing of longer amplicons allows for the detection of rare monoallelic double and triple mutations of value in the cases of tumor heterogeneity. KRAS double mutants have previously been identified, indicating that tumor heterogeneity is possible in certain cancer types $(13,14)$. Further, the primary PCR amplification of the region of interest can be used as the template for the subsequent FTSS and FASS reactions as well as SBE reactions. Thus, the robust, simple work flow of SBE (with $1 \%$ detection level) can be coupled to CE-based FTSS for the identification of unknown mutations, with the analysis as a fragment file increasing the limit of detection and reducing problematic peak under peak detection that can occur when analyzed as a sequencing file (Figure 1). Further, combining the fragment analysis approach with selective amplification methods such as COLD-PCR and ice-COLD PCR would further improve the limit of detection for FTSS and FASS reactions $(15,16)$.

\section{Acknowledgments}

Thomas Ingalls for assistance in growing cell cultures.

\section{Competing interests}

C.D., E.Z., M-P. G., F.W., J.B., and E.S. are employees of Life Technologies. Life Technologies products referred to within this publication are currently For Research Use Only. Not intended for animal or human therapeutic or diagnostic use.

\section{References}

1. Karnoub, A.E. and R.A. Weinberg. 2008. Ras oncogenes: split personalities. Nat. Rev. Mol. Cell Biol. 9:517-531.

2. Minamoto, T., M. Mai, and Z. Ronai. 2000. K-ras mutation: early detection in molecular diagnosis and risk assessment of colorectal, pancreas, and lung cancers--a review. Cancer Detect. Prev. 24:1-12.

3. Lièvre, A., J.B. Bachet, D. Le Corre, V. Boige, B. Landi, J.F. Emile, J.F. Côté, G. Tomasic, et al. 2006. KRAS mutation status is predictive of response to cetuximab therapy in colorectal cancer. Cancer Res. 66:3992-3995.

4. Amado, R.G., M. Wolf, M. Peeters, E. Van Cutsem, S. Siena, D.J. Freeman, T. Juan, R. Sikorski, et al. 2008. Wild-type KRAS is required for panitumumab efficacy in patients with metastatic colorectal cancer. J. Clin. Oncol. 26:1626-1634.

5. van Krieken, J.H., A. Jung, T. Kirchner, F, Carneiro, R. Seruca, F.T. Bosman, P. Quirke, J.F. Fléjou, et al. 2008. KRAS mutation testing for predicting response to anti-EGFR therapy for colorectal carcinoma: proposal for an European quality assurance program. Virchows Arch. 453:417-431.

6. Pao, W., T.Y. Wang, G.J. Riely, V.A. Miller, Q. Pan, M. Ladanyi, M.F. Zakowski, R.T. Heelan, et al. 2005. KRAS mutations and primary resistance of lung adenocarcinomas to gefitinib or erlotinib. PLoS Med. 2:e17.

7. Shackelford, W., S. Deng, K. Murayama, and J. Wang. 2004. A new technology for mutation detection. Ann. N. Y. Acad. Sci. 1022:257262.

8. Di Fiore, F., F. Blanchard, F. Charbonnier, F. Le Pessot, A. Lamy, M.P. Galais, L. Bastit, A. Killian, et al. 2007. Clinical relevance of KRAS mutation detection in metastatic colorectal cancer treated by Cetuximab plus chemotherapy. Br. J. Cancer 96:1166-1169.

9. Magnin, S., E. Viel, A. Baraquin, S. ValmaryDegano, B. Kantelip, J.L. Pretet, C. Mougin, M. Bigand, et al. 2011. A multiplex SNaPshot assay as a rapid method for detecting KRAS and BRAF mutations in advanced colorectal cancers. J. Mol. Diagn. 13:485-492.

10. Ogino, S., T. Kawasaki, M. Brahmandam, L. Yan, M. Cantor, C. Namgyal, M. MinoKenudson, G.Y. Lauwers, et al. 2005. Sensitive sequencing method for KRAS mutation detection by Pyrosequencing J. Mol. Diagn. 7:413-421.

11. Simi, L., N. Pratesi, M. Vignoli, R. Sestini, F. Cianchi, R. Valanzano, S. Nobili, E. Mini, et al. 2008. High-resolution melting analysis for rapid detection of KRAS, BRAF, and PIK3CA gene mutations in colorectal cancer. Am. J. Clin. Pathol. 130:247-253.

12. Franklin, W.A., J. Haney, M. Sugita, L. Bemis, A. Jimeno, and W.A. Messersmith. 2010. KRAS mutation: comparison of testing methods and tissue sampling techniques in colon cancer. J. Mol. Diagn. 12:43-50.

13. Tsiatis, A.C., A. Norris-Kirby, R.G. Roy, M.J. Hafez, C.D. Gocke, J.R. Eshleman, and K.M. Murphy. 2010. Comparison of Sanger Sequencing, Pyrosequencing, and Melting Curve Analysis for the Detection of KRAS Mutations. J. Mol. Diagn. 12:425-432.

14. Oliner, K., T. Juan, S. Suggs, M. Wolf, I. Sarosi, D.J. Freeman, T. Gyuris, W. Baron, et al. 2010. A comparability study of 5 commercial KRAS tests. Diagn. Pathol. 16:23.

15. Li, J., L. Wang, H. Mamon, M.H. Kulke, R. Berbeco, and G.M. Makrigiorgos. 2008 Replacing PCR with COLD-PCR enriches variant DNA sequences and redefines the sensitivity of genetic testing. Nat. Med. 14:579584 .

16. Milbury, C.A., J. Li, and G.M. Makrigiorgos. 2011. Ice-COLD-PCR enables rapid amplification and robust enrichment for low-abundance unknown DNA mutations. Nucleic Acids Res. 39:e2.

Received 4 January 2012; accepted 27 July 2012.

Address correspondence to Colin Davidson, Life Technologies, 850 Lincoln Centre Drive, Foster City, CA, USA. Email: colin.davidson@lifetech. com

Supplementary material for this article is available at www.BioTechniques.com/article/113913

To purchase reprints of this article, contact: biotechniques@fosterprinting.com 